\title{
Nurses and opioids: results of a bi-national survey on mental models regarding opioid administration in hospitals
}

This article was published in the following Dove Press journal:

Journal of Pain Research

I March 2017

Number of times this article has been viewed

\author{
Charlotte Guest' \\ Fabian Sobotka ${ }^{2}$ \\ Athina Karavasopoulou ${ }^{3}$ \\ Stephen Ward ${ }^{3}$ \\ Carsten Bantel ${ }^{4,5}$ \\ 'Pain Medicine, Chelsea and \\ Westminster Hospital NHS \\ Foundation Trust, London, UK; \\ ${ }^{2}$ Division of Epidemiology and \\ Biometry, Department of Health \\ Services Research, Faculty 6, Medicine \\ and Health Sciences, Carl von \\ Ossietzky Universität Oldenburg, \\ Oldenburg, Germany; '3Pain Service, \\ Barts Health, St Bartholomew's \\ Hospital, London, UK; ${ }^{4}$ Department \\ of Anaesthesiology, Intensive Care, \\ Emergency Medicine and Pain Therapy, \\ Oldenburg University, Klinikum \\ Oldenburg Campus, Oldenburg, \\ Germany; ${ }^{5}$ Department of Surgery \\ and Cancer, Anaesthetics Section, \\ Imperial College London, Chelsea \\ and Westminster Hospital Campus, \\ London, UK
}

Correspondence: Carsten Bantel Department of Anaesthesiology, Intensive Care, Emergency Medicine and Pain Therapy, Oldenburg University, Klinikum Oldenburg Campus, Rahel-Straus-Strasse 10, 26133 Oldenburg, Germany

Tel +49 44I 40377173

Email carsten.bantel@uni-oldenburg.de
Objective: Pain remains insufficiently treated in hospitals. Increasing evidence suggests human factors contribute to this, due to nurses failing to administer opioids. This behavior might be the consequence of nurses' mental models about opioids. As personal experience and conceptions shape these models, the aim of this prospective survey was to identify model-influencing factors.

Material and methods: A questionnaire was developed comprising of 14 statements concerning ideations about opioids and seven questions concerning demographics, indicators of adult learning, and strength of religious beliefs. Latent variables that may underlie nurses' mental models were identified using undirected graphical dependence models. Representative items of latent variables were employed for ordinal regression analysis. Questionnaires were distributed to 1,379 nurses in two London, UK, hospitals $(n=580)$ and one German $(n=799)$ hospital between September 2014 and February 2015.

Results: A total of 511 (37.1\%) questionnaires were returned. Mean (standard deviation) age of participants were 37 (11) years; $83.5 \%$ participants were female; $45.2 \%$ worked in critical care; and $51.5 \%$ had more than 10 years experience. Of the nurses, $84 \%$ were not scared of opioids, $87 \%$ did not regard opioids as drugs to help patients die, and $72 \%$ did not view them as drugs of abuse. More English (41\%) than German (28\%) nurses were afraid of criminal investigations and were constantly aware of side effects (UK, 94\%; Germany, 38\%) when using opioids. Four latent variables were identified which likely influence nurses' mental models: "conscious decision-making"; "medication-related fears"; "practice-based observations"; and "risk assessment". They were predicted by strength of religious beliefs and indicators of informal learning such as experience but not by indicators of formal learning such as conference attendance.

Conclusion: Nurses in both countries employ analytical and affective mental models when administering the opioids and seem to learn from experience rather than from formal teaching. Additionally, some attitudes and emotions towards opioids are likely the result of nurses' cultural background.

Keywords: nurses, opioids, mental models, decision-making

\section{Introduction}

Despite considerable awareness and the introduction of practice recommendations, pain remains frequently under-treated in hospitals. ${ }^{1-4}$ Although the limited pharmacological choices are usually considered responsible, the classification of pain as a vital sign acknowledged the human factor as a cause for treatment failures as well. ${ }^{5}$ The idea of staff-related barriers in pain management is further supported by evidence suggesting nurses often either fail to administer opioids or fail to identify the correct dose. ${ }^{6-8}$ Medication errors are repeatedly attributed to a lack of education. ${ }^{9}$ However, evidence indicates pain management does not improve after nurses have been subjected to teaching, suggesting other 
influences may also contribute. ${ }^{10} \mathrm{McCaffery}$ et al, for instance, found personal opinions influenced how nurses' titrated and administered opioids. ${ }^{11}$ We thus hypothesize opioid administration by nurses is partly a consequence of their mental models. Mental models refer to a phenomenon (here, opioid administration) and include "personally constructed internal conceptions that affect how a person acts" and makes decisions. ${ }^{12}$ As these models are developed through subjective interpretations of experiences, they can be expected to be influenced by cultural and social factors such as country of origin, religion, type of training, or exposure to news media. The first objective of this study was hence to explore such influences and how they might interact with nurses' mental models about opioids. The second aim was to identify universally valid aspects of these mental models. For this purpose a prospective cross-sectional questionnaire-based study was conducted in two distinctively different cohorts. The first cohort was recruited from London, UK, hospitals with their culturally diverse and academically trained nursing staff. ${ }^{13,14}$ The second was enrolled from ethnically more homogenous but less academically trained staff of a northern German hospital. ${ }^{15,16}$

\section{Methods}

The study was conducted at three centers in two countries: in London, UK, at Chelsea and Westminster Hospital (CW) and St Bartholomew's Hospital (Bart's); in Oldenburg, Germany, at Klinikum Oldenburg Hospital. All centers are teaching hospitals providing care for all medical specialties.

The study was approved and registered as service evaluation with the research and development departments of the two London hospitals; reference numbers, 1097 (CW) and 5477 (Bart's), therefore not requiring ethical review according to English regulations. In Germany the study was waived by the local ethics committee (Oldenburg University, Drs.85/2014).

\section{Questionnaire development}

To explore nurses' mental models about opioids a questionnaire was developed as follows.

Following written informed consent, semi-structured interviews were conducted with $n=6$ nurses of different specialties and levels of seniority. Responses were tape-recorded and transcribed. Transcripts were analyzed for recurrent themes. Themes were listed, compared, and formed the basis for $n=55$ preliminary statements. Content and face validity of each statement was assessed using 5-point Likert scales and free comments to rate each statement. Assessments were completed by $\mathrm{n}=7$ pain experts, including doctors, nurses, physiotherapists, and psychologists. Statements rating poorly were removed leaving $n=20$ items for an initial questionnaire. This was piloted on $n=10$ nurses. After entering responses into a database (SPSS version 22, IBM, Portsmouth, UK) a correlation matrix was generated. Unrelated items ( $r$-values $<0.3$ ) indicating latent variables unconnected to the research question and highly related items ( $r$-values $>0.8)$ indicating item redundancies were excluded. ${ }^{17}$ Subsequently, $n=6$ statements were removed leaving the final questionnaire with $n=14$ items. Questionnaire responses were recorded employing 5-point Likert scales from "strongly agree" to "strongly disagree" (Figure S1).

\section{Predictor variables}

Seven variables potentially influencing participants' responses were included. These comprised participant demographics, indicators of adult learning, strength of religious beliefs, and the medical specialty they worked in (Figure S1). Specialty comprised medicine, surgery, and critical care, which included nurses working in intensive care, recovery and emergency departments.

Three types of adult learning can be distinguished: formal, non-formal, and informal. ${ }^{18}$ Formal learning refers to structured programs typically concluded by examinations. ${ }^{18}$ Achieving a nursing degree is the result of formal learning at universities in UK and at non-academic facilities in Germany. ${ }^{19}$ Therefore, school education and academic degrees were a priori different between cohorts and consequently not assessed here.

Non-formal learning is structured but not assessed. Conferences are its prototypical formats in medicine. ${ }^{18,20,21}$ Thus a question related to conference attendance was included. Finally, informal learning is the most common form of learning. It is part of everyday life hence unstructured and relying on reflecting experiences (experiential learning). ${ }^{18,22}$ Questions concerning nurses' specialties (workplace experience), number of years qualified, and frequency of following the daily news assessed this.

According to the 2012 Pew report, 75\% of the English and German population, felt affiliated to a religion. ${ }^{23}$ This suggests religion may influence nurses' mental models. This influence might differ with the degree of religiousness. Therefore, a question concerning participants' subjective strength of religious beliefs was included.

\section{Translation}

For use in Germany, the questionnaire was translated into German by two native Germans and one native English speaker fluent in German. 


\section{Data acquisition}

Between September 2014 and February 2015 all n=1,379 registered nurses working in the participating hospitals were invited to volunteer. They were asked to individually complete the questionnaire and return it anonymously to the study lead of each site.

\section{Statistical analysis}

Data were entered into a database (SPSS version 22) and analyzed descriptively. Where appropriate, responses obtained on a Likert scale were grouped as follows: "strongly agree" and "agree" pooled into an "agree" group and "strongly disagree" and "disagree" into a "disagree" group.

Associations between the 14 item responses were estimated using undirected graphical dependence models incorporating conditional independences between items. Each item was represented as a node and every association as an edge between two nodes. The final model was generated through stepwise elimination of weak associations (edges). After each step the line with the smallest calculated $\gamma$-coefficient was removed and the process repeated until the conditioned independence of one additional line was not included in the $95 \%$ confidence interval.

The variability of the final model was estimated through 1000 replications of a non-parametric bootstrap, which according to Pigeot represents the variability of the entire model. ${ }^{24}$ Associations that determined the final model were identified through averaging the bootstrap. The final model contained a complete set of sub-graphs for each group of dependent items, which allowed categorization of item groups according to content themes (latent variables). Graphical inspection of the item groups enabled identification of "central" items with the most and strongest connections to others. Those central items were determined for each group and used to investigate the potential influence of predictors on the latent variables.

Ordinal regression was first employed to identify predictors for similar and dissimilar responses between cohorts and then for representative items of latent variables. Important covariables were determined through stepwise backward elimination using the Akaike information criterion (AIC). Initial analysis was performed for the pooled data of both cohorts including "country" as a covariable. However, in the final step it was repeated independently for each country.

Data analysis was performed with " $R$ " version 3.1.2 (The $\mathrm{R}$ Foundation for Statistical Computing, Vienna, Austria). A $p<0.05$ was considered significant.

\section{Results}

A total of 511 (37.1\%) questionnaires were returned with overall $1.5 \%$ data missing (Tables 1 and 2). Characteristics of participants are shown in Table 1. The majority of nurses in both groups were female and on average 36 to 39 years-of-age.

English participants worked more frequently in critical care but had less experience compared to their German colleagues. They also attended conferences more often and had stronger religious beliefs (Table 1).

\section{Distribution of responses and predictor variables}

Overall reliability of the questionnaire was good (Cronbach's $\alpha: 0.80$ ).

Distribution of responses including missing data for each item is shown in Table 2. Similar response patterns between cohorts were found for items 4, 5, 8, 9, 11 and 13. Nurses

Table I Characteristics of participants

\begin{tabular}{|c|c|c|c|}
\hline & Total & UK & Germany \\
\hline Nurses approached n (\%) & $1379(100)$ & $580(100)$ & $799(100)$ \\
\hline Forms returned n (\%) & $511(37.1)$ & $284(49.0)$ & $227(28.4)$ \\
\hline Age (years) mean (SD)* & $37(\mathrm{II})$ & $36(10)$ & 39 (II) \\
\hline \multicolumn{4}{|l|}{ Gender } \\
\hline Female: $\mathrm{n}(\%)$ & $427(83.5)$ & $238(83.8)$ & $189(83.3)$ \\
\hline Male: n (\%) & $80(15.7)$ & $45(15.8)$ & $35(15.4)$ \\
\hline Missing: $n(\%)$ & $4(0.8)$ & $\mathrm{I}(0.4)$ & $3(1.3)$ \\
\hline \multicolumn{4}{|l|}{ Specialty } \\
\hline Medicine: $\mathrm{n}(\%)$ & $157(30.7)$ & $75(26.4)$ & $82(36.1)$ \\
\hline Surgery: n (\%) & $103(20.2)$ & $49(17.3)$ & $54(23.8)$ \\
\hline Critical Care*: n (\%) & $231(45.2)$ & $145(5 \mid .0)$ & $86(37.9)$ \\
\hline Missing: n (\%) & $20(3.9)$ & $15(5.3)$ & $5(2.2)$ \\
\hline \multicolumn{4}{|l|}{ Years qualified } \\
\hline $0-2:$ n (\%) & $81(15.8)$ & $63(22.2)$ & $18(7.9)$ \\
\hline $2-5: \mathrm{n}(\%)$ & $74(14.5)$ & $49(17.3)$ & $25(I I .0)$ \\
\hline 5-10: n (\%) & $89(17.4)$ & $52(18.3)$ & $37(16.3)$ \\
\hline$>10: \mathrm{n}(\%)$ & $263(51.5)$ & $118(4 \mid .5)$ & 145 (63.9) \\
\hline Missing: n (\%) & $4(0.8)$ & $2(0.7)$ & $2(0.9)$ \\
\hline \multicolumn{4}{|l|}{ Following daily news } \\
\hline Frequently: n (\%) & $393(76.9)$ & $218(76.7)$ & $175(77.0)$ \\
\hline Occasionally: n (\%) & $97(19.0)$ & $58(20.4)$ & $39(17.2)$ \\
\hline Rarely/never: n (\%) & $16(3.1)$ & $7(2.5)$ & $9(4.0)$ \\
\hline Missing: n (\%) & $5(1.0)$ & I (0.4) & $4(1.8)$ \\
\hline \multicolumn{4}{|l|}{ Conference attendance } \\
\hline Frequently: n (\%) & $190(37.1)$ & $120(42.3)$ & $70(30.8)$ \\
\hline Occasionally: n (\%) & $261(51.1)$ & $140(49.3)$ & $12 \mid(53.3)$ \\
\hline Rarely/never: n (\%) & $56(11.0)$ & $22(7.7)$ & $34(15.0)$ \\
\hline Missing: n (\%) & $4(0.8)$ & $2(0.7)$ & $2(0.9)$ \\
\hline \multicolumn{4}{|l|}{ Strong religious beliefs } \\
\hline Agreement: $\mathrm{n}(\%)$ & $154(30.1)$ & $116(40.8)$ & $38(16.7)$ \\
\hline Undecided: n (\%) & $107(20.9)$ & $64(22.5)$ & $43(18.9)$ \\
\hline Disagreement: n (\%) & $221(43.3)$ & $88(31.1)$ & $133(58.6)$ \\
\hline Prefer not to answer & $24(4.7)$ & $14(4.9)$ & $10(4.4)$ \\
\hline Missing: n (\%) & $5(1.0)$ & $2(0.7)$ & $3(1.3)$ \\
\hline
\end{tabular}

Notes: *Critical Care comprises nurses working in Intensive Care Unit-High Dependency Unit settings, Recovery and Accident and Emergency departments. Abbreviations: SD, standard deviation. 
Table 2 Frequency distribution of nurses' responses

\begin{tabular}{|c|c|c|c|c|c|c|c|c|c|c|c|c|}
\hline & \multicolumn{4}{|l|}{ Total } & \multicolumn{4}{|l|}{ UK } & \multicolumn{4}{|c|}{ Germany } \\
\hline & $\bar{A}$ & $\mathbf{U}$ & D & $\begin{array}{l}\text { Missing } \\
\text { data }\end{array}$ & $\bar{A}$ & $\mathbf{U}$ & D & $\begin{array}{l}\text { Missing } \\
\text { data }\end{array}$ & $\bar{A}$ & $\mathbf{U}$ & D & $\begin{array}{l}\text { Missing } \\
\text { data }\end{array}$ \\
\hline $\begin{array}{l}\text { I. The huge variety of new opioids } \\
\text { available makes administration more } \\
\text { difficult }\end{array}$ & $\begin{array}{l}125 \\
(24.5)\end{array}$ & $\begin{array}{l}163 \\
(31.9)\end{array}$ & $\begin{array}{l}213 \\
(41.7)\end{array}$ & $\begin{array}{l}10 \\
(2.0)\end{array}$ & $\begin{array}{l}65 \\
(22.9)\end{array}$ & $\begin{array}{l}79 \\
(27.8)\end{array}$ & $\begin{array}{l}136 \\
(47.9)\end{array}$ & $\begin{array}{l}4 \\
(1.4)\end{array}$ & $\begin{array}{l}60 \\
(26.4)\end{array}$ & $\begin{array}{l}84 \\
(37.0)\end{array}$ & $\begin{array}{l}77 \\
(33.9)\end{array}$ & $\begin{array}{l}6 \\
(2.6)\end{array}$ \\
\hline $\begin{array}{l}\text { 2. I require more knowledge about } \\
\text { opioids (eg, morphine) compared } \\
\text { to other medications (eg, blood } \\
\text { pressure medications or insulin) in } \\
\text { order to give them safely. }\end{array}$ & $\begin{array}{l}232 \\
(45.4)\end{array}$ & $\begin{array}{l}94 \\
(18.4)\end{array}$ & $\begin{array}{l}178 \\
(34.8)\end{array}$ & $\begin{array}{l}7 \\
(1.4)\end{array}$ & $\begin{array}{l}104 \\
(36.6)\end{array}$ & $\begin{array}{l}48 \\
(16.9)\end{array}$ & $\begin{array}{l}130 \\
(45.8)\end{array}$ & $\begin{array}{l}2 \\
(0.7)\end{array}$ & $\begin{array}{l}128 \\
(56.4)\end{array}$ & $\begin{array}{l}46 \\
(20.3)\end{array}$ & $\begin{array}{l}48 \\
(2 I . I)\end{array}$ & $\begin{array}{l}5 \\
(2.2)\end{array}$ \\
\hline $\begin{array}{l}\text { 3. When giving opioids (eg, morphine) } \\
\text { I need to monitor patients more } \\
\text { closely in comparison to giving other } \\
\text { medications (eg, blood pressure } \\
\text { medications or insulin). }\end{array}$ & $\begin{array}{l}266 \\
(52.1)\end{array}$ & $\begin{array}{l}120 \\
(23.5)\end{array}$ & $\begin{array}{l}119 \\
(23.3)\end{array}$ & $\begin{array}{l}6 \\
(1.2)\end{array}$ & $\begin{array}{l}165 \\
(58.1)\end{array}$ & $\begin{array}{l}54 \\
(19.0)\end{array}$ & $\begin{array}{l}61 \\
(21.5)\end{array}$ & $\begin{array}{l}4 \\
(1.4)\end{array}$ & $\begin{array}{l}101 \\
(44.5)\end{array}$ & $\begin{array}{l}66 \\
(29.1)\end{array}$ & $\begin{array}{l}58 \\
(25.6)\end{array}$ & $\begin{array}{l}2 \\
(0.9)\end{array}$ \\
\hline $\begin{array}{l}\text { 4. Opioids (eg, morphine) scare me } \\
\text { which means I am less likely to want } \\
\text { to administer them. }\end{array}$ & $\begin{array}{l}22 \\
(4.3)\end{array}$ & $\begin{array}{l}56 \\
(11.0)\end{array}$ & $\begin{array}{l}431 \\
(84.3)\end{array}$ & $\begin{array}{l}2 \\
(0.4)\end{array}$ & $\begin{array}{l}14 \\
(4.9)\end{array}$ & $\begin{array}{l}27 \\
(9.5)\end{array}$ & $\begin{array}{l}242 \\
(85.2)\end{array}$ & $\begin{array}{l}\text { I } \\
(0.4)\end{array}$ & $\begin{array}{l}8 \\
(3.5)\end{array}$ & $\begin{array}{l}29 \\
(12.8)\end{array}$ & $\begin{array}{l}189 \\
(83.3)\end{array}$ & $\begin{array}{l}\text { I } \\
(0.4)\end{array}$ \\
\hline $\begin{array}{l}\text { 5. When giving opioids (eg, morphine) } \\
\text { I am afraid of overdose. }\end{array}$ & $\begin{array}{l}89 \\
(17.4)\end{array}$ & $\begin{array}{l}110 \\
(21.5)\end{array}$ & $\begin{array}{l}310 \\
(60.7)\end{array}$ & $\begin{array}{l}2 \\
(0.4)\end{array}$ & $\begin{array}{l}68 \\
(23.9)\end{array}$ & $\begin{array}{l}59 \\
(20.8)\end{array}$ & $\begin{array}{l}156 \\
(54.9)\end{array}$ & $\begin{array}{l}\text { I } \\
(0.4)\end{array}$ & $\begin{array}{l}21 \\
(9.3)\end{array}$ & $\begin{array}{l}51 \\
(22.5)\end{array}$ & $\begin{array}{l}154 \\
(67.8)\end{array}$ & $\begin{array}{l}\text { I } \\
(0.4)\end{array}$ \\
\hline $\begin{array}{l}\text { 6. Prescribing errors are a common } \\
\text { barrier to nurses administering } \\
\text { opioids (eg, morphine). }\end{array}$ & $\begin{array}{l}129 \\
(25.2)\end{array}$ & $\begin{array}{l}117 \\
(22.9)\end{array}$ & $\begin{array}{l}252 \\
(49.3)\end{array}$ & $\begin{array}{l}13 \\
(2.5)\end{array}$ & $\begin{array}{l}100 \\
(35.2)\end{array}$ & $\begin{array}{l}66 \\
(23.2)\end{array}$ & $\begin{array}{l}113 \\
(39.8)\end{array}$ & $\begin{array}{l}5 \\
(1.8)\end{array}$ & $\begin{array}{l}29 \\
(12.8)\end{array}$ & $\begin{array}{l}51 \\
(22.5)\end{array}$ & $\begin{array}{l}139 \\
(61.2)\end{array}$ & $\begin{array}{l}8 \\
(3.5)\end{array}$ \\
\hline $\begin{array}{l}\text { 7. Opioids (eg, morphine) are } \\
\text { dangerous because they are } \\
\text { controlled drugs and require double } \\
\text { signing. }\end{array}$ & $\begin{array}{l}149 \\
(29.2)\end{array}$ & $\begin{array}{l}102 \\
(20.0)\end{array}$ & $\begin{array}{l}248 \\
(48.5)\end{array}$ & $\begin{array}{l}12 \\
(2.3)\end{array}$ & $\begin{array}{l}96 \\
(33.8)\end{array}$ & $\begin{array}{l}56 \\
(19.7)\end{array}$ & $\begin{array}{l}127 \\
(44.7)\end{array}$ & $\begin{array}{l}5 \\
(1.8)\end{array}$ & $\begin{array}{l}53 \\
(23.3)\end{array}$ & $\begin{array}{l}46 \\
(20.3)\end{array}$ & $\begin{array}{l}12 \mid \\
(53.3)\end{array}$ & $\begin{array}{l}7 \\
(3.1)\end{array}$ \\
\hline $\begin{array}{l}\text { 8. Nurses often associate giving opioids } \\
\text { (eg, morphine) with helping patients } \\
\text { to die. }\end{array}$ & $\begin{array}{l}24 \\
(4.7)\end{array}$ & $\begin{array}{l}39 \\
(7.6)\end{array}$ & $\begin{array}{l}444 \\
(86.9)\end{array}$ & $\begin{array}{l}4 \\
(0.8)\end{array}$ & $\begin{array}{l}19 \\
(6.7)\end{array}$ & $\begin{array}{l}28 \\
(9.9)\end{array}$ & $\begin{array}{l}235 \\
(82.7)\end{array}$ & $\begin{array}{l}2 \\
(0.7)\end{array}$ & $\begin{array}{l}5 \\
(2.2)\end{array}$ & $\begin{array}{l}11 \\
(4.8)\end{array}$ & $\begin{array}{l}209 \\
(92.1)\end{array}$ & $\begin{array}{l}2 \\
(0.9)\end{array}$ \\
\hline $\begin{array}{l}\text { 9. Familiarity with an opioid (eg, } \\
\text { morphine) gives me more confidence } \\
\text { when administering this opioid. }\end{array}$ & $\begin{array}{l}435 \\
(85.1)\end{array}$ & $\begin{array}{l}48 \\
(9.4)\end{array}$ & $\begin{array}{l}23 \\
(4.5)\end{array}$ & $\begin{array}{l}5 \\
(1.0)\end{array}$ & $\begin{array}{l}255 \\
(89.8)\end{array}$ & $\begin{array}{l}15 \\
(5.3)\end{array}$ & $\begin{array}{l}12 \\
(4.2)\end{array}$ & $\begin{array}{l}2 \\
(0.7)\end{array}$ & $\begin{array}{l}180 \\
(79.3)\end{array}$ & $\begin{array}{l}33 \\
(14.5)\end{array}$ & $\begin{array}{l}11 \\
(4.8)\end{array}$ & $\begin{array}{l}3 \\
(1.3)\end{array}$ \\
\hline $\begin{array}{l}\text { 10. When giving opioids (eg, morphine) } \\
\text { I am constantly aware of side effects. }\end{array}$ & $\begin{array}{l}352 \\
(68.9)\end{array}$ & $\begin{array}{l}75 \\
(14.7)\end{array}$ & $\begin{array}{l}80 \\
(15.7)\end{array}$ & $\begin{array}{l}4 \\
(0.8)\end{array}$ & $\begin{array}{l}266 \\
(93.7)\end{array}$ & $\begin{array}{l}11 \\
(3.9)\end{array}$ & $\begin{array}{l}4 \\
(1.4)\end{array}$ & $\begin{array}{l}3 \\
(1.1)\end{array}$ & $\begin{array}{l}86 \\
(37.9)\end{array}$ & $\begin{array}{l}64 \\
(28.2)\end{array}$ & $\begin{array}{l}76 \\
(33.5)\end{array}$ & $\begin{array}{l}\text { I } \\
(0.4)\end{array}$ \\
\hline $\begin{array}{l}\text { II. Nurses associate opioids } \\
\text { (eg, morphine) with drug abuse. }\end{array}$ & $\begin{array}{l}50 \\
(9.8)\end{array}$ & $\begin{array}{l}89 \\
(17.4)\end{array}$ & $\begin{array}{l}366 \\
(71.6)\end{array}$ & $\begin{array}{l}6 \\
(1.2)\end{array}$ & $\begin{array}{l}38 \\
(13.4)\end{array}$ & $\begin{array}{l}65 \\
(22.9)\end{array}$ & $\begin{array}{l}177 \\
(62.3)\end{array}$ & $\begin{array}{l}4 \\
(1.4)\end{array}$ & $\begin{array}{l}12 \\
(5.3)\end{array}$ & $\begin{array}{l}24 \\
(10.6)\end{array}$ & $\begin{array}{l}189 \\
(83.3)\end{array}$ & $\begin{array}{l}2 \\
(0.9)\end{array}$ \\
\hline $\begin{array}{l}\text { 12. When using opioids (eg, morphine) } \\
\text { I don't want to make mistakes } \\
\text { because I am afraid of criminal } \\
\text { investigations. }\end{array}$ & $\begin{array}{l}180 \\
(35.2)\end{array}$ & $\begin{array}{l}109 \\
(21.3)\end{array}$ & $\begin{array}{l}209 \\
(40.9)\end{array}$ & $\begin{array}{l}13 \\
(2.5)\end{array}$ & $\begin{array}{l}116 \\
(40.8)\end{array}$ & $\begin{array}{l}56 \\
(19.7)\end{array}$ & $\begin{array}{l}101 \\
(35.6)\end{array}$ & $\begin{array}{l}\text { II } \\
(3.9)\end{array}$ & $\begin{array}{l}64 \\
(28.2)\end{array}$ & $\begin{array}{l}53 \\
(23.3)\end{array}$ & $\begin{array}{l}108 \\
(47.6)\end{array}$ & $\begin{array}{l}2 \\
(0.9)\end{array}$ \\
\hline $\begin{array}{l}\text { 13. When administering opioids } \\
\text { (eg, morphine) I am more concerned } \\
\text { about patients with a history of drug } \\
\text { abuse (IVDU). }\end{array}$ & $\begin{array}{l}286 \\
(56.0)\end{array}$ & $\begin{array}{l}122 \\
(23.9)\end{array}$ & $\begin{array}{l}90 \\
(17.6)\end{array}$ & $\begin{array}{l}13 \\
(2.5)\end{array}$ & $\begin{array}{l}154 \\
(54.2)\end{array}$ & $\begin{array}{l}66 \\
(23.2)\end{array}$ & $\begin{array}{l}54 \\
(19.0)\end{array}$ & $\begin{array}{l}10 \\
(3.5)\end{array}$ & $\begin{array}{l}132 \\
(58.1)\end{array}$ & $\begin{array}{l}56 \\
(24.7)\end{array}$ & $\begin{array}{l}36 \\
(15.9)\end{array}$ & $\begin{array}{l}3 \\
(1.3)\end{array}$ \\
\hline $\begin{array}{l}\text { 14. I need to trust the prescribing doctor } \\
\text { in order to be comfortable with } \\
\text { giving an opioid (eg, morphine). }\end{array}$ & $\begin{array}{l}280 \\
(54.8)\end{array}$ & $\begin{array}{l}101 \\
(19.8)\end{array}$ & $\begin{array}{l}118 \\
(23.1)\end{array}$ & $\begin{array}{l}12 \\
(2.3)\end{array}$ & $\begin{array}{l}130 \\
(45.8)\end{array}$ & $\begin{array}{l}64 \\
(22.5)\end{array}$ & $\begin{array}{l}81 \\
(28.5)\end{array}$ & $\begin{array}{l}9 \\
(3.2)\end{array}$ & $\begin{array}{l}150 \\
(66.1)\end{array}$ & $\begin{array}{l}37 \\
(16.3)\end{array}$ & $\begin{array}{l}37 \\
(16.3)\end{array}$ & $\begin{array}{l}3 \\
(1.3)\end{array}$ \\
\hline
\end{tabular}

Notes: Response data are shown as $\mathrm{n}(\%)$.

Abbreviations: A, agreement; U, undecided; D, disagreement; IVDU: intravenous drug use.

in both countries disagreed with statements 4 (opioids scare me), 5 (when giving opioids I am afraid of overdose), 8 (nurses associate giving opioids with helping patients to die) and 11 (nurses associate opioids with drug abuse). However, nurses agreed with statements 9 (familiarity with an opioid gives me more confidence when administering it) and 13 (when administering opioids I am more concerned about patients with a history of drug abuse).

Regression analysis for items with similar responses in both cohorts identified six potential predictors (Table 3A). "Strong religious beliefs" (items 5, 8, 11, 13) and "following daily news" (items $5,11,13$ ) were the most common. 
Table 3 Results of ordinal regression analysis - similarities and differences of items between countries

\begin{tabular}{|c|c|c|c|c|c|c|c|}
\hline \multicolumn{8}{|c|}{ A. Predictors of items that show a similar trend in UK and Germany } \\
\hline \multicolumn{4}{|c|}{$\begin{array}{l}\text { Item 4: Opioids (eg, morphine) scare me which means I am } \\
\text { less likely to want to administer them. }\end{array}$} & \multicolumn{4}{|c|}{$\begin{array}{l}\text { Item 5: When giving opioids (eg, morphine) I am afraid of } \\
\text { overdose. }\end{array}$} \\
\hline Predictor & Coefficient & SE & p-value & Predictor & Coefficient & SE & $p$-value \\
\hline \multicolumn{4}{|c|}{ Specialty (reference: medicine) } & \multicolumn{4}{|c|}{ Gender (reference: male) } \\
\hline Surgery & 1.02 & 0.35 & 0.003 & Female & 0.46 & 0.29 & 0.111 \\
\hline \multirow[t]{11}{*}{ Critical Care } & 0.18 & 0.33 & 0.575 & \multicolumn{4}{|c|}{ Years qualified (reference: $0-2$ years) } \\
\hline & & & & $2-5$ years & -0.70 & 0.33 & 0.033 \\
\hline & & & & $5-10$ years & -0.84 & 0.33 & 0.011 \\
\hline & & & & $>10$ years & -1.04 & 0.27 & 0.000 \\
\hline & & & & \multicolumn{4}{|c|}{ Following daily news (reference: rarely/ never) } \\
\hline & & & & Occasionally & -0.96 & 0.54 & 0.074 \\
\hline & & & & Frequently & -1.02 & 0.50 & 0.042 \\
\hline & & & & \multicolumn{4}{|c|}{ Strong religious beliefs (reference: prefer not to answer) } \\
\hline & & & & Disagreement & -0.54 & 0.46 & 0.239 \\
\hline & & & & Undecided & -0.41 & 0.48 & 0.400 \\
\hline & & & & Agreement & 0.16 & 0.47 & 0.730 \\
\hline \multicolumn{4}{|c|}{$\begin{array}{l}\text { Item 8: Nurses often associate giving opioids (eg, morphine) } \\
\text { with helping patients to die. }\end{array}$} & \multicolumn{4}{|c|}{$\begin{array}{l}\text { Item 9: Familiarity with an opioid (eg, morphine) gives me } \\
\text { more confidence when administering this opioid. }\end{array}$} \\
\hline Predictor & Coefficient & SE & $p$-value & Predictor & Coefficient & SE & $p$-value \\
\hline \multicolumn{4}{|c|}{ Strong religious beliefs (reference: prefer not to answer) } & Age & & & \\
\hline Disagreement & -0.93 & 0.62 & 0.136 & Age & -0.03 & 0.01 & 0.015 \\
\hline Undecided & -0.76 & 0.66 & 0.251 & & & & \\
\hline Agreement & 0.10 & 0.60 & 0.870 & & & & \\
\hline \multicolumn{4}{|c|}{$\begin{array}{l}\text { Item I I: Nurses associate opioids (eg, morphine) with drug } \\
\text { abuse. }\end{array}$} & \multicolumn{4}{|c|}{$\begin{array}{l}\text { Item I3: When administering opioids (eg, morphine) I am more } \\
\text { concerned about patients with a history of drug abuse (IVDU). }\end{array}$} \\
\hline Predictor & coefficient & SE & p-value & Predictor & coefficient & SE & p-value \\
\hline \multicolumn{4}{|l|}{ Age } & \multicolumn{4}{|c|}{ Specialty (reference: medicine) } \\
\hline Age & 0.04 & 0.02 & 0.025 & Surgery & 0.42 & 0.28 & 0.130 \\
\hline \multicolumn{4}{|c|}{ Years qualified (reference: $0-2$ years) } & Critical Care & -0.37 & 0.22 & 0.087 \\
\hline $2-5$ years & -0.85 & 0.40 & 0.035 & & & & \\
\hline $5-10$ years & -0.51 & 0.37 & 0.165 & & & & \\
\hline$>10$ years & -0.90 & 0.40 & 0.026 & & & & \\
\hline \multicolumn{4}{|c|}{ Following daily news (reference: rarely/ never) } & \multicolumn{4}{|c|}{ Following daily news (reference: rarely/ never) } \\
\hline Occasionally & -0.33 & 0.59 & 0.579 & Occasionally & 0.17 & 0.56 & 0.757 \\
\hline Frequently & -0.96 & 0.56 & 0.087 & Frequently & -0.43 & 0.52 & 0.409 \\
\hline \multicolumn{4}{|c|}{ Strong religious beliefs (reference: prefer not to answer) } & \multicolumn{4}{|c|}{ Strong religious beliefs (reference: prefer not to answer) } \\
\hline Disagreement & -0.64 & 0.52 & 0.224 & Disagreement & -1.04 & 0.51 & 0.042 \\
\hline Undecided & -0.65 & 0.56 & 0.242 & Undecided & -0.87 & 0.53 & 0.102 \\
\hline Agreement & 0.44 & 0.52 & 0.396 & Agreement & -0.49 & 0.52 & 0.345 \\
\hline
\end{tabular}

\section{B. Predictors of items that show a dissimilar trend in UK and Germany}

Item 2: I require more knowledge about opioids $(\mathrm{eg}$, morphine) compared to other medications (eg, blood pressure medications or insulin) in order to give them safely.

\begin{tabular}{|c|c|c|c|c|c|c|c|}
\hline Predictor & Coefficient & SE & $p$-value & Predictor & Coefficient & SE & $p$-value \\
\hline \multicolumn{4}{|c|}{ Specialty (reference: medicine) } & \multicolumn{4}{|c|}{ Specialty (reference: medicine) } \\
\hline Surgery & 0.21 & 0.27 & 0.446 & Surgery & 0.08 & 0.26 & 0.754 \\
\hline Critical Care & -0.28 & 0.22 & 0.196 & Critical Care & -0.53 & 0.23 & 0.021 \\
\hline \multicolumn{4}{|c|}{ Years qualified (reference: $0-2$ years) } & \multicolumn{4}{|c|}{ Strong religious beliefs (reference: prefer not to answer) } \\
\hline $2-5$ years & -1.00 & 0.34 & 0.003 & Disagreement & -0.33 & 0.46 & 0.475 \\
\hline $5-10$ years & $-1.3 \mid$ & 0.33 & 0.000 & Undecided & 0.17 & 0.48 & 0.723 \\
\hline$>10$ years & -0.92 & 0.28 & 0.001 & Agreement & 0.64 & 0.47 & 0.172 \\
\hline \multicolumn{8}{|c|}{ Conference attendance (reference: rarely/ never) } \\
\hline Occasionally & 0.17 & 0.31 & 0.578 & & & & \\
\hline Frequently & -0.24 & 0.32 & 0.442 & & & & \\
\hline \multicolumn{4}{|c|}{ Country (reference: UK) } & \multicolumn{4}{|c|}{ Country (reference: UK) } \\
\hline Germany & 1.38 & 0.25 & 0.000 & Germany & -0.99 & 0.24 & 0.000 \\
\hline
\end{tabular}

(Continued)
Item 6: Prescribing errors are a common barrier to nurses administering opioids (eg, morphine). 
Table 3 (Continued)

\begin{tabular}{|c|c|c|c|c|}
\hline \multicolumn{5}{|c|}{ B. Predictors of items that show a dissimilar trend in UK and Germany } \\
\hline \multicolumn{4}{|c|}{$\begin{array}{l}\text { Item I0: When giving opioids (eg, morphine) I am constantly } \\
\text { aware of side effects. }\end{array}$} & \multirow[t]{2}{*}{ No item } \\
\hline Predictor & Coefficient & SE & p-value & \\
\hline \multicolumn{5}{|l|}{ Age } \\
\hline Age & -0.03 & 0.01 & 0.022 & \\
\hline \multicolumn{5}{|c|}{ Specialty (reference: medicine) } \\
\hline Surgery & 0.02 & 0.31 & 0.947 & \\
\hline Critical Care & 0.63 & 0.28 & 0.026 & \\
\hline \multicolumn{5}{|c|}{ Following daily news (reference: rarely/ never) } \\
\hline Occasionally & -0.23 & 0.63 & 0.719 & \\
\hline Frequently & 0.50 & 0.59 & 0.398 & \\
\hline \multicolumn{5}{|c|}{ Country (reference: UK) } \\
\hline Germany & -3.13 & 0.32 & 0.000 & \\
\hline
\end{tabular}

Notes: For regression analysis responses to items were coded as I=disagree, $2=$ neither agree/disagree, $3=$ agree. For easier orientation $p$-values $<0.05$ are shown in bold. Abbreviations: SE, standard error; IVDU: intravenous drug use.

Dissimilar responses between cohorts were identified for items 2 (I require more knowledge about opioids compared to other medications in order to give them safely), 6 (prescribing errors are a common barrier to nurses administering opioids), and 10 (when giving opioids I am constantly aware of side effects). Responses to item 10 showed $93.7 \%$ of English nurses agreed, compared to $37.9 \%$ in the German cohort (Table 2). "Country" was a significant predictor for these three items (Table 3B). Among the remaining possible predictors "specialty" was the most important.

\section{Latent variables}

Graphical models were built for both cohorts (Figure 1). A global system of connections allowed the differentiation of sub-networks representing distinct latent variables. Four latent variables were identified and labelled as "conscious decision-making"; "medication-related fears"; "practice-based observations"; and "risk assessment". For each sub-network one central item was determined and used as representative dependent variable in subsequent regression analyses (Table 4).

Graphical models and predictor profiles revealed differences and similarities between cohorts (Table 4). "Conscious decision-making" was predicted by "years qualified" in the English and "specialty" plus "following daily news" in the German cohort. "Specialty" predicted "medication-related fears" in the UK whereas "strong religious beliefs" predicted "medication-related fears" in Germany. Further, "practicebased observations" was predicted by "years qualified", "specialty" and "strong religious beliefs" in the English cohort and by "specialty" in Germany. Because of the equal strengths of connections between items, no representative statement was identified and hence no predictor analysis performed for "risk assessment" in the English cohort. In Germany "risk assessment" was predicted by "specialty".
A

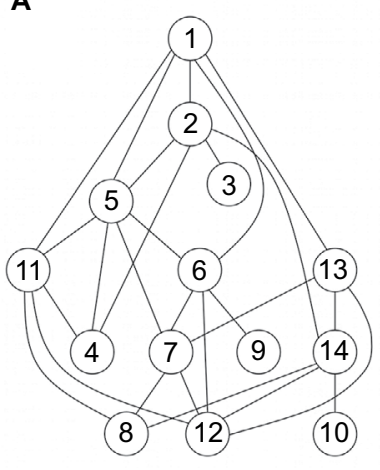

B

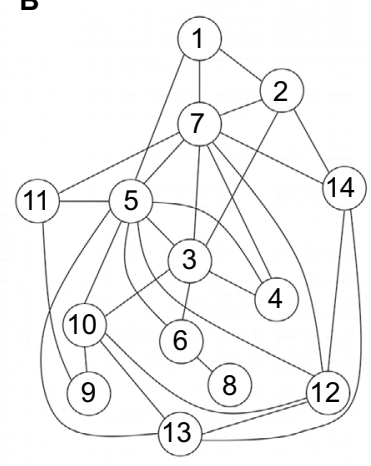

Figure I Graphical models of associations between items. (A) English cohort; (B) German cohort.

Notes: Numbers in circles represent the respective items from the questionnaire. Lines indicate how items are connected with each other. Visual inspection allowed identification of 'central items' for each cohort which were subsequently used for regression analysis.

\section{Discussion}

Pain management in hospitals remains suboptimal. ${ }^{1,3}$ Among other factors, nurses' mental models about opioids may contribute. For instance, "medication-related fears" or misguided "decision-making" may hinder drug administration. Surprisingly, little is known regarding nurse-related factors affecting opioid application. This study was the first to investigate and compare nurses' mental models about opioids in two countries. Results indicated participants were predominantly guided by four underlying principles; "conscious decision-making"; "medication-related fears"; "practice-based observations"; and "risk assessment". Additionally, responses showed some interesting similarities and differences between cohorts.

\section{Similar responses}

Most English and German nurses were not afraid of using opioids, nor were they anxious about overdosing. These 
Table 4 Results of ordinal regression analysis - predictors of latent variables

\begin{tabular}{|c|c|c|c|c|c|c|c|}
\hline \multicolumn{4}{|c|}{ A. UK } & \multicolumn{4}{|l|}{ B. Germany } \\
\hline \multicolumn{4}{|c|}{ Conscious decision making } & \multicolumn{4}{|c|}{ Conscious decision making } \\
\hline \multicolumn{4}{|c|}{$\begin{array}{l}\text { Item 5: When giving opioids (eg, morphine) I am afraid of } \\
\text { overdose. }\end{array}$} & \multicolumn{4}{|c|}{$\begin{array}{l}\text { Item 5: When giving opioids (eg, morphine) I am afraid of } \\
\text { overdose. }\end{array}$} \\
\hline Predictor & Coefficient & SE & $p$-value & Predictor & Coefficient & SE & $p$-value \\
\hline \multicolumn{4}{|c|}{ Years qualified (reference: $0-2$ years) } & \multicolumn{4}{|c|}{ Gender (reference: male) } \\
\hline $2-5$ years & -0.91 & 0.40 & 0.022 & Female & 0.82 & 0.49 & 0.093 \\
\hline $5-10$ years & -1.09 & 0.41 & 0.008 & & & & \\
\hline$>10$ years & -1.08 & 0.34 & 0.001 & & & & \\
\hline \multicolumn{4}{|c|}{ Conference attendance (reference: rarely/ never) } & \multicolumn{4}{|c|}{ Specialty (reference: medicine) } \\
\hline Occasionally & -0.05 & 0.45 & 0.908 & Surgery & 0.83 & 0.39 & 0.032 \\
\hline Frequently & -0.62 & 0.47 & 0.184 & Critical Care & 0.32 & 0.35 & 0.364 \\
\hline \multicolumn{4}{|c|}{ Strong religious beliefs (reference: prefer not to answer) } & \multicolumn{4}{|c|}{ Following daily news (reference: rarely/never) } \\
\hline Disagreement & -0.45 & 0.68 & 0.510 & Occasionally & -1.90 & 0.76 & 0.012 \\
\hline Undecided & -0.59 & 0.70 & 0.404 & Frequently & -1.87 & 0.69 & 0.007 \\
\hline Agreement & 0.55 & 0.65 & 0.404 & & & & \\
\hline \multicolumn{4}{|c|}{ Medication related fears } & \multicolumn{4}{|c|}{ Medication related fears } \\
\hline \multicolumn{4}{|c|}{$\begin{array}{l}\text { Item I4: I need to trust the prescribing doctor in order to be } \\
\text { comfortable with giving an opioid (eg, morphine). }\end{array}$} & \multicolumn{4}{|c|}{$\begin{array}{l}\text { Item 7: Opioids (eg, morphine) are dangerous because they } \\
\text { are controlled drugs and require double signing. }\end{array}$} \\
\hline Predictor & Coefficient & SE & $p$-value & Predictor & Coefficient & SE & $p$-value \\
\hline \multicolumn{8}{|c|}{ Specialty (reference: medicine) } \\
\hline Surgery & -0.02 & 0.39 & 0.951 & & & & \\
\hline Critical Care & -0.62 & 0.31 & 0.043 & & & & \\
\hline \multicolumn{8}{|c|}{ Conference attendance (reference: rarely/ never) } \\
\hline Occasionally & 0.89 & 0.48 & 0.062 & & & & \\
\hline Frequently & 0.51 & 0.48 & 0.284 & & & & \\
\hline \multicolumn{4}{|c|}{ Strong religious beliefs (reference: prefer not to answer) } & \multicolumn{4}{|c|}{ Strong religious beliefs (reference: prefer not to answer) } \\
\hline Disagreement & -1.16 & 0.62 & 0.060 & Disagreement & -1.11 & 0.69 & 0.106 \\
\hline Undecided & -1.02 & 0.64 & 0.112 & Undecided & -1.12 & 0.74 & 0.132 \\
\hline Agreement & -0.59 & 0.61 & 0.339 & Agreement & -1.89 & 0.76 & 0.013 \\
\hline \multicolumn{4}{|c|}{ Practice based observations } & Practice based & titions & & \\
\hline $\begin{array}{l}\text { Item 6: Prescril } \\
\text { administering o }\end{array}$ & $\begin{array}{l}\text { ors are a com } \\
\text { eg, morphine }\end{array}$ & barrie & o nurses & $\begin{array}{l}\text { Item 3: When } \\
\text { monitor patien } \\
\text { medications (e }\end{array}$ & $\begin{array}{l}\text { pioids (eg, mo } \\
\text { closely in co } \\
\text { pressure me }\end{array}$ & $\begin{array}{l}\text { e) I n } \\
\text { son tc } \\
\text { ons or }\end{array}$ & $\begin{array}{l}\text { to } \\
\text { ing other } \\
\text { ulin). }\end{array}$ \\
\hline Predictor & Coefficient & SE & $p$-value & Predictor & Coefficient & SE & $p$-value \\
\hline Years qualified ( $r$ & $0-2$ years) & & & Age & & & \\
\hline $2-5$ years & -0.23 & 0.39 & 0.555 & Age & -0.02 & 0.01 & 0.152 \\
\hline $5-10$ years & -1.12 & 0.41 & 0.007 & & & & \\
\hline$>10$ years & -0.37 & 0.34 & 0.272 & & & & \\
\hline Specialty (referen & cine) & & & Gender (referenc & & & \\
\hline Surgery & -0.38 & 0.38 & 0.318 & Female & -0.68 & 0.38 & 0.071 \\
\hline Critical Care & -0.94 & 0.32 & 0.003 & & & & \\
\hline Strong religious $b$ & ference: prefer & answe & & Specialty (referen & cine) & & \\
\hline Disagreement & -1.38 & 0.64 & 0.031 & Surgery & 1.00 & 0.35 & 0.005 \\
\hline Undecided & -0.60 & 0.63 & $0.34 I$ & Critical Care & 0.48 & 0.31 & 0.117 \\
\hline Agreement & 0.08 & 0.62 & 0.895 & & & & \\
\hline Risk assessmen & & & & Risk assessmen & & & \\
\hline No item & & & & $\begin{array}{l}\text { Item I 2: When } \\
\text { make mistakes }\end{array}$ & $\begin{array}{l}\text { pioids (eg, } \mathrm{m} \\
\text { e I am afraid }\end{array}$ & $\begin{array}{l}\text { e) I d } \\
\text { ninal }\end{array}$ & $\begin{array}{l}\text { want to } \\
\text { stigations. }\end{array}$ \\
\hline & & & & Predictor & Coefficient & SE & $p$-value \\
\hline & & & & Specialty (referen & cine) & & \\
\hline & & & & Surgery & 0.67 & 0.33 & 0.044 \\
\hline & & & & Critical Care & 0.14 & 0.31 & 0.659 \\
\hline
\end{tabular}

Notes: For regression analysis responses to items were coded as $I=$ disagree, $2=$ neither agree/disagree, $3=$ agree. For easier orientation $p$-values $<0.05$ are shown in bold. Abbreviations: SE, standard error. 
responses were identified more often in experienced nurses or those working in areas with high opioid usage (critical care) suggesting knowledge about opioids contributes to a stressfree routine. This was further supported by nurses being more confident about an opioid when they were familiar with it. Results hence support Briggs, who argues training in pain management should closely link fact-learning with acquisition of hands-on experience. ${ }^{25}$

Furthermore, nurses in both cohorts displayed considerable ambivalence concerning opioids and drug misuse. Although nurses were worried about using opioids in patients with a history of substance misuse, they did not associate opioids with drug abuse. This ambivalence was reminiscent of Broekmans et al's work showing opioid-addiction is viewed by nurses as a mere side-effect rather than a medical condition. ${ }^{26}$ As reasons for these inconsistencies remain speculative, our results suggest experience, working in critical care and possibly following the news may help diminish concerns about opioids.

Interestingly, despite opioids being commonly used in palliative care in both countries, the majority of nurses did not view them as a mean to help patients dying. ${ }^{27,28}$ Regardless of the frequent controversial public debates about assisted dying responses were not influenced by "following the daily news". ${ }^{29,30}$ Most nurses therefore understand the mainstay of opioids is to alleviate pain and suffering. In support of Berghs et al they therefore showed a high level of professionalism regarding the use of opioids. ${ }^{31}$

\section{Different responses}

Most English but only one-third of German nurses appeared to be aware of side-effects when administering opioids. Observing such differences concerning drug safety was surprising. It might indicate a lack of general understanding about opioids, appropriate doses and administration intervals by German nurses. This notion was supported by a higher number of German participants agreeing they needed more knowledge to safely administer opioids; and to trust the prescriber. In addition, they also disagreed more often that prescribing errors were barriers to opioid administration.

The potential knowledge gap regarding opioids might be a consequence of nursing training in Germany. A recent European survey and a German governmental report suggested that the German training system might need improving; emphasizing higher academic levels for nurses in other European countries. ${ }^{15,32}$ The report also criticized a worse climate for training in Germany and positively highlighted the self-governing body for UK nurses to organize and regulate professional education. ${ }^{15}$

\section{Mental models - latent variables}

The four latent variables ("conscious-decision making"; "medication-related fears"; "practice-based observations"; "risk assessment") identified in this study to underlie nurses' mental models about opioids are in accordance with the "dual theory of information processing". ${ }^{33}$ This theory distinguishes an "experiential" and "rational" system. ${ }^{34}$ The experiential system (system 1) is automatic and affective whereas the rational system (system 2 ) is conscious and logical. Although system 2 can construct and organize thoughts and is capable of overriding system 1 , it is nevertheless system 1 that creates beliefs and choices. ${ }^{34}$

"Medication-related fears" and "practice-based observations" are traditionally regarded belonging to system $1 .{ }^{35,36}$ Although in this study "medication-related fears" were influenced by the strength of religious beliefs, this differed between cohorts. Whether a characteristic of a specific faith or the greater cultural diversity in London compared to northern Germany influenced this, needs future exploration. ${ }^{13,14,16}$ Additionally, findings such as "conference attendance" and "specialty" mitigating fears in the English cohort re-emphasized the importance of continuous adult learning. The value of learning was also supported by the identification of "years qualified" and "specialty" as predictors for "practice-based observations". "Practice-based observations" in this study was synonymous with "experiential learning", the learning that relies on practice and obtaining feedback. ${ }^{21,22,37}$ It was hence not surprising that experienced nurses and nurses frequently using opioids learned more as they had a greater chance to interact with colleagues, their biggest source of information. ${ }^{38,39}$

Results therefore suggest, in order to shape mental models more effectively, nurses should acquire an archive of opioidrelated events by working in an environment with high exposure and be able to share their experiences with others. ${ }^{11,40}$

"Conscious decision-making" is commonly viewed as a rational act. ${ }^{41}$ Yet, studies indicate it is complex, likely also involving affective processes. ${ }^{42,43}$ This was noted here as well. Although neither variables with strong rational (conference attendance) or emotional (religious beliefs) connotations were found to be predictors, the predictive values of indicators of experiential learning nevertheless supported the idea. This notion is based on the increasing recognition of experiential learning as an emotional and rational process..$^{39,44}$ The interplay of emotion and rationality during decision-making was further supported in the German cohort through the influence of "following the daily news", which is also rational and emotive. ${ }^{45,46}$ 
"Risk assessment", intrinsically linked to decisionmaking, is normally regarded as rational. ${ }^{35,47}$ However, Slovic et al suggest it might be affective, too. ${ }^{35,43}$ Affect in risk assessment is common in situations of uncertainty which are frequent in medicine. ${ }^{48-50}$ It was noted here as well, as a quarter of nurses viewed having larger choices of opioids disadvantageous, possibly because it reduces familiarity and confidence. Additionally, nearly half of all participants felt opioid use required more knowledge than administering other medications. These data hence suggest it might be better to limit the number of opioid-medications to allow nurses to gain thorough experience with them.

\section{Important predictors}

Unsurprisingly, learning was identified as key predictor of nurses' mental models, hence highlighting the importance of continued education in pain medicine.$^{51}$ However, our results also suggest learning contexts need further exploration and possibly modification. This notion is based on previous data and our findings indicating experiential (years qualified and specialty) and other types of informal learning (following the news) were more important than formal learning (conference attendance)..$^{52}$

"Following the news" can affect people differently. For instance, the media might help self-reflection, improve problem solving, enhance social skills or facilitate learning through employment of short memorable messages. ${ }^{53-57}$

However, media can also exert negative influences as information may be biased or incomplete. ${ }^{58}$ In addition, Schmidt et al showed frequent misdiagnoses when doctors were exposed to disease-related information via the media prior to reviewing patients. ${ }^{59}$ Further, Dasgupta et al found news coverage of opioids preceded increased rates of medication overdose. ${ }^{60}$ Therefore the true value of media exposure for self-directed learning remains elusive. Nevertheless, this study suggests positive influences as nurses frequently following the news were less concerned about opioid overdose or misuse.

"Strength of religious beliefs" also predicted nurses' mental models. Religious beliefs in the context of healthcare are usually judged in terms of patient outcomes, as there is evidence they can assist prevention and treatment of diseases. ${ }^{61-63}$ Religion may nevertheless exert negative effects, for instance, prohibiting treatments. ${ }^{62}$ Additionally, it might affect patients' opinions about opioids as taking analgesics might be regarded as violating divine will. ${ }^{64-67}$

As religion can influence patients, it may also impact nurses' practice. Interestingly, only few studies have investigated this yet. ${ }^{31,68}$ Moreover, data suggest nurses are not aware their beliefs might affect their work. ${ }^{68}$
This study supports the idea of religiosity influencing pain management. Nurses with strong beliefs were more anxious of overdosing and employing opioids for patients with a history of drug misuse. They were also more likely to view opioids as means of assisted dying or drugs of abuse.

Our observations suggest "not doing harm" was more important for religious than non-religious participants. This supported Berghs et al's findings that religious nurses were more likely to reject euthanasia. ${ }^{31}$ However, how this mind-set translates into practice warrants further research.

\section{Study limitations}

A limitation was the relatively low participation rate in Germany. However, low return rates are common in this type of research but results are nevertheless regarded as valid. ${ }^{69,70}$ This is supported here by the identification of similar response patterns to six items in both cohorts. It is unlikely further recruitment would have considerably changed results. Even where dissimilar responses were found, trends were clear and unlikely to have benefited from more participation.

Also, the high proportion of participants working in critical care might have biased results. Still, $50 \%$ of nurses were recruited from other specialties, leaving enough variability and hence information in the data to draw valid conclusions. Although future research should aim for a more even subgroup distribution, this might be difficult to achieve in questionnaire-based studies.

Furthermore, questionnaire-based research is often hampered by participants only able to respond to fixed statements. ${ }^{71}$ However, this questionnaire was closer to "real life" issues as it was based on nurse interviews. Yet, future research should reinterview participants and observe their practice.

Finally, assessment of nurses' religiosity could also be criticized. However, as "religion" and "religiosity" are multifaceted concepts a more detailed approach was beyond the scope of this study. Nevertheless, because religion was considered in this study, it already satisfied Levin's call for research on the influence of religion on attitudes, beliefs, and experience in healthcare. ${ }^{61}$

\section{Conclusion}

Results suggest when nurses use opioids, they employ both analytical and affective mental models to make decisions. Their attitudes towards opioids can be considered professional since opioids were viewed as analgesics rather than substances of abuse or medications to assist dying. Further, nurses seem to be influenced by cultural background, and they learn more from experience than from formal teaching. 


\section{Acknowledgments}

The authors would like to thank Dr Susan Childs for her comments on the questionnaires as well as Dr Bianca Kuehler and Rebecca Kuehler for their help in the translation process.

\section{Author Contributions}

C Guest and C Bantel designed the study and conducted the pilot experiments. F Sobotka did the statistical analysis. C Guest, C Bantel, A Karavasopoulou, and S Ward collected the data. All authors contributed toward data analysis, drafting and revising the paper and agree to be accountable for all aspects of the work.

\section{Disclosure}

Carsten Bantel has received funding for consultancy work for Mundipharma. The other authors report no conflicts of interest in this work.

\section{References}

1. Sommer M, de Rijke JM, van Kleer M, et al. The prevalence of postoperative pain in a sample of 1490 surgical inpatients. Eur J Anaesthesiol. 2008;25(4):267-274.

2. Apfelbaum JL, Chen C, Mehta SS, Gan TJ. Postoperative pain experience: results from a national survey suggest postoperative pain continues to be undermanaged. Anesth Analg. 2003;97(2):534-540.

3. King AC, Kuo PY, Bantel C, editors. A day of pain - results of a snapshot audit in pain management from a major teaching hospital. In: Wells JCD, editor. 15th World Congress of Pain Clinicians; 2012 June 27-30; Granada, Spain. Bologna: Medimond; 2012:45-50.

4. Faculty of Pain Medicine of the Royal College of Anaesthetists. Core Standards for Pain Management Services in the UK. 2015. Available from http://www.rcoa.ac.uk/system/files/FPM-CSPMS-UK2015.pdf. Accessed 10 November, 2016.

5. Geriatrics and Extended Care Strategic Healthcare Group, National Pain Management Coordinating Committee. Pain as the 5th vital sign toolkit. 2000. Department of Vertrans Affairs, Washington, DC, USA. http://www.va.gov/PAINMANAGEMENT/docs/Pain_As_the_5th_ Vital_Sign_Toolkit.pdf. Accessed November 10, 2016.

6. Symons VC, McMurray A. Factors influencing nurses to withhold surgical patients" oral medications pre- and postoperatively. Collegian. 2014;21(4):267-274.

7. Murnion BP, Gnjidic D, Hilmer SN. Prescription and administration of opioids to hospital in-patients, and barriers to effective use. Pain Med. 2010;11(1):58-66.

8. Kane-Gill SL, Kowiatek JG, Weber RJ. A comparison of voluntarily reported medication errors in intensive care and general care units. Qual Saf Health Care. 2010;19(1):55-59.

9. McCaffery M, Pasero C, Ferrell B. Nurses' Decisions About Opioid Dose. Am J Nurs. 2007;107(2):35-39.

10. Ene KW, Nordberg G, Bergh I, Johansson FG, Sjostrom B. Postoperative pain management - the influence of surgical ward nurses. J Clin Nurs. 2008;17(15):2042-2050.

11. McCaffery M, Ferrell BR, Pasero C. Nurses' personal opinions about patients" pain and their effect on recorded assessments and titration of opioid doses. Pain Manag Nurs. 2000;1(3):79-87.

12. Rook L. Mental models: a robust definition. The Learning Organization. 2013;20(1):38-47.

13. Wood P, Landry C, Bloomfield J. Cultural diversity in Britain: A toolkit for cross-cultural co-operation. York: Comedia \& The Joseph Rowntree Foundation. 2006. Available from https://www.jrf.org.uk/sites/default/ files/jrf/migrated/files/1922-cultural-diversity-britain.pdf. Accessed November 10, 2016.
14. Jivraj S. How has ethnic diversity grown 1991-2001-2011? Manchester: Centre on Dynamics of Ethnicity (CoDE). 2012. Available from http:// www.ethnicity.ac.uk/medialibrary/briefings/dynamicsofdiversity/howhas-ethnic-diversity-grown-1991-2001-2011.pdf. Accessed November $10,2016$.

15. Bundesministerium für Forschung und Bildung. Bestandsaufnahme der Ausbildung in den Gesundheitsfachberufen im europäischen Vergleich. [Federal Ministry for Research and Education. Comprehensive training in health care professions in a European comparison]. 2014. Available from https://www.bmbf.de/pub/berufsbildungsforschung_band_15.pdf. Accessed November 10, 2016. German.

16. Kahle S, Püschel O. Die Metropolregion Bremen-Oldenburg - Ausgewählte Ergebnisse des Zensus 2011. [The Metropolitan region Bremen-Oldenburg - Selected results of the Census 2011]. Bremen: Statistisches Landesamt. 2014. Available from www.statistik.niedersachsen.de/download/83755. Accessed November 10, 2016. German.

17. Field A. Exploratory factor analysis. In: Field A, editor. Discovering Statistics Using SPSS. London: Sage; 2009:627-685.

18. Colardyn D, Bjornavold J. Validation of Formal, Non-Formal and Informal Learning: policy and practices in EU Member States. European Journal of Education. 2004;39(1):27.

19. Robinson S, Griffiths P. Nursing education and regulation: international profiles and perspectives. London: King's College London. 2007. Available from http://www.kcl.ac.uk/nursing/research/nnru/Publications/ Reports/NurseEduProfiles.pdf. Accessed November 10, 2016.

20. Nývlt O. Stastistical Survey of Non-Formal Education. Statistika. 2012;49(4):27-34.

21. van de Wiel MW, Van den Bossche P, Janssen S, Jossberger H. Exploring deliberate practice in medicine: how do physicians learn in the workplace? Adv Health Sci Educ Theory Pract. 2011;16(1):81-95.

22. Fowler J. Experiential learning and its facilitation. Nurse Educ Today. 2008;28(4):427-433.

23. Hackett C, Grim BJ. The Global Religious Landscape - A Report on the Size and Distribution of the World's Major Religious Groups as of 2010. Washington: Pew Research Center's Forum on Religion \& Public Life; 2012. Available from http://www.pewforum.org/files/2014/01/ global-religion-full.pdf. Accessed November 11, 2016.

24. Pigeot I, Sobotka F, Kreiner S, Foraita R. The uncertainty of a selected graphical model. J Appl Stat. 2015;42(11):2335-2352.

25. Briggs EV, Carr EC, Whittaker MS. Survey of undergraduate pain curricula for healthcare professionals in the United Kingdom. Eur J Pain. 2011;15(8):789-795.

26. Broekmans S, Vanderschueren S, Morlion B, Kumar A, Evers G. Nurses' attitudes toward pain treatment with opioids: a survey in a Belgian university hospital. Int J Nurs Stud. 2004;41(2):183-189.

27. Engeser P, Hermann K, Szecsenyi J, Peters-Klimm F. Symptom control and place of death in palliative cancer patients in primary care. Results of the controlled PAMINO evaluation study. Schmerz. 2014;28(6): $607-613$.

28. Neuberger J, Guthrie C, Aaronovitch D, et al. More Care, Less Pathway A Review of the Liverpool Care Pathway. United Kingdom Government; 2013:1-63. Available from https://www.gov.uk/government/uploads/ system/uploads/attachment_data/file/212450/Liverpool_Care_Pathway. pdf. Accessed November 9, 2016.

29. Khaleeli H, Cocozza P. Assisted dying bill: "I want to control how and when I die". The Guardian. 2014 July 17; Available from https://www. theguardian.com/society/2014/jul/16/assisted-dying-people-strugglingterminal-illness. Accessed November 9, 2016.

30. Drobinski M. Suche nach dem guten Tod. [Search for the good death] Süddeutsche Zeitung. 2015 July 2. Available from http://www.sueddeutsche.de/politik/sterbehilfe-vom-guten-tod-1.2545782. Accessed November 9, 2016. German.

31. Berghs M, Dierckx de Casterle B, Gastmans C. The complexity of nurses' attitudes toward euthanasia: a review of the literature. $J$ Med Ethics. 2005;31(8):441-446.

32. Aiken LH, Sloane DM, Bruyneel L, Van den Heede K, Sermeus W, Consortium RC. Nurses' reports of working conditions and hospital quality of care in 12 countries in Europe. Int J Nurs Stud. 2013;50(2):143-153. 
33. Epstein $\mathrm{S}$. Integration of the cognitive and the psychodynamic unconscious. Am Psychol. 1994;49(8):709-724.

34. Kahneman D. Two Systems. In: Kahneman D, editor. Thinking, Fast and Slow. London: Penguin Books; 2012:17-106.

35. Slovic P, Finucane ML, Peters E, MacGregor DG. Risk as analysis and risk as feelings: some thoughts about affect, reason, risk, and rationality. Risk Anal. 2004;24(2):311-322.

36. Kahneman D, Klein G. Conditions for intuitive expertise: a failure to disagree. Am Psychol. 2009;64(6):515-526.

37. Bunniss S, Kelly DR. "The unknown becomes the known": collective learning and change in primary care teams. Med Educ. 2008;42(12): 1185-1194.

38. Doran DM, Mylopoulos J, Kushniruk A, et al. Evidence in the palm of your hand: development of an outcomes-focused knowledge translation intervention. Worldviews Evid Based Nurs. 2007;4(2):69-77.

39. Wagter JM, van de Bunt G, Honing M, Eckenhausen M, Scherpbier A. Informal interprofessional learning: visualizing the clinical workplace. $J$ Interprof Care. 2012;26(3):173-182.

40. Cranley L, Doran DM, Tourangeau AE, Kushniruk A, Nagle L. Nurses' uncertainty in decision-making: a literature review. Worldviews Evid Based Nurs. 2009;6(1):3-15.

41. Rottenstreich YS, Kivetz R. On decision making without likelihood judgment. Organ Behav Hum Dec. 2006;101:74-88.

42. Zajonc RB. Feeling and Thinking - Preferences Need No Inferences Am Psychol. 1980;35(2):151-175.

43. Slovic P, Peters E, Finucane ML, Macgregor DG. Affect, risk, and decision making. Health Psychol. 2005;24(4 Suppl):S35-40.

44. Felten P, Gilchrist LZ, Darby A. Emotion and Learning: Feeling our Way Toward a New Theory of Reflection in Service-learning. Michigan Journal of Community Service Learning. 2006;12(2):38-46.

45. Ackerman PL, Kanfer R, Calderwood C. Use it or lose it? Wii brain exercise practice and reading for domain knowledge. Psychol Aging. 2010;25(4):753-766.

46. Igartua J-J, Moral-Toranzo F, Fernandez I. Cognitive, Attitudinal, and Emotional Effects of News Frame and Group Cues, on Processing News about Immigration. J Media Psychol. 2011;23(4):174-185.

47. Kemshall H, Wilkinson B, Baker K. Skills for Risk Assessment. In: Kemshall H, Wilkinson B, Baker K, editors. Working with Risk. Cambridge: Polity Press; 2013:9-42.

48. Suchman AL. Uncertainty, competence, and opioids. J Gen Intern Med. 2005;20(6):554-555.

49. Cioffi J, Markham R. Clinical decision-making by midwives: managing case complexity. J Adv Nurs. 1997;25(2):265-272.

50. French B. Uncertainty and information need in nursing. Nurse Educ Today. 2006;26(3):245-252.

51. Eraut M. Non-formal learning and tacit knowledge in professional work. Br J Educ Psychol. 2000;70(Pt 1):113-136.

52. Davis D. Does CME work? An analysis of the effect of educational activities on physician performance or health care outcomes. Int $J$ Psychiatry Med. 1998;28(1):21-39.
53. Rochon PA, Bero LA, Bay AM, et al. Comparison of review articles published in peer-reviewed and throwaway journals. JAMA. 2002;287(21): 2853-2856.

54. McDonnell PJ. The role of tabloids in ophthalmic education: pro. Arch Ophthalmol. 2004;122(9):1379-1380.

55. Kirkpatrick MK. NINE: Newspapers in Nursing Education. Nurse Educ. 1994;19(6):21-23.

56. Stanovich KE, Cunningham AE. Where does knowledge come from? Specific associations between print exposure and information aquisition. J Educ Psychol. 1993;85(2):211-229.

57. Jeffres LW, Lee J-w, Neuendorf K, Atkin D. Newspaper reading supports community involvement. Newsp Res J. 2007;28(1):6-23.

58. Prosser H, Clayson K. A content analysis of prescription drug information in the UK print news media. Int J Pharm Pract. 2008(16) 223-230.

59. Schmidt HG, Mamede S, Van den Berge K, Van Gog T, Van Sasse JLCM, Rikers RMJP. Exposure to media information about a disease can cause doctors to misdiagnose similar-looking clinical cases. Acad Med. 2014;89(2):285-291.

60. Dasgupta N, Mandl KD, Brownstein JS. Breaking the news or fueling the epidemic? Temporal association between news media report volume and opioid-related mortality. PLoS One. 2009;4(11):e7758.

61. Levin J. Religion and mental health: Theory and research. International Journal of Applied Psychoanalytic Studies 2010:1-14.

62. Koenig HG. Religion, spirituality, and medicine: research findings and implications for clinical practice. South Med J. 2004;97(12):1194-1200.

63. Ellison CG, Levin JS. The religion-health connection: evidence, theory, and future directions. Health Educ Behav. 1998;25(6):700-720.

64. Bosch F, Banos JE. Religious beliefs of patients and caregivers as a barrier to the pharmacologic control of cancer pain. Clin Pharmacol Ther. 2002;72(2):107-111.

65. O'Rourke K. Pain relief: the perspective of Catholic tradition. J Pain Symptom Manage. 1992;7(8):485-491.

66. Fitzpatrick SJ, Kerridge IH, Jordens CF, et al. Religious Perspectives on Human Suffering: Implications for Medicine and Bioethics. J Relig Health. 2016;55(1):159-173.

67. Long A. Nursing: a spiritual perspective. Nurs Ethics. 1997;4(6): 496-510.

68. Latour JM, Fulbrook P, Albarran JW. EfCCNa survey: European intensive care nurses' attitudes and beliefs towards end-of-life care. Nurs Crit Care. 2009;14(3):110-121.

69. Pack-Mabien A, Labbe E, Herbert D, Haynes J Jr. Nurses' attitudes and practices in sickle cell pain management. Appl Nurs Res. 2001;14(4): 187-192.

70. Weenink JW, Westert GP, Schoonhoven L, Wollersheim H, Kool RB. Am I my brother's keeper? A survey of 10 healthcare professions in the Netherlands about experiences with impaired and incompetent colleagues. BMJ Qual Saf. 2015;24(1):56-64.

71. Long SO. Life is more than a survey: understanding attitudes toward euthanasia in Japan. Theor Med Bioeth. 2002;23(4-5):305-319. 


\section{Supplementary material}

Thank you for taking the time to complete this questionnaire in order to help me with my dissertation. The aim of my dissertation is to explore post-registration nurses' views on administering opioids.

Demographics:

Age: years

Gender: (Please circle one) Male Female

Speciality: (Please circle one) Medicine Surgery Emergency/Acute Assessment

Recovery ICU/HDU/Burns

Number of Years Qualified? (Please circle one)
$0-2$ years
$2-5$ years
$5-10$ years
10 years+

Qualification: (Please circle one)

Diploma Degree Masters $\quad \mathrm{PhD}$

I keep up-to-date with the news: (Please circle one)

Very Frequently Frequently Occasionally Rarely Very Rarely Never

I attend conferences/study days/courses: (Please circle one)

Very Frequently Frequently Occasionally Rarely Very Rarely Never

I have strong religious beliefs: (Please circle one)

Strongly Agree $\quad$ Agree Neither agree nor disagree Disagree $\quad$ Strongly Disagree

Prefer not to answer

Please circle one answer for each question below:

Q1. The huge variety of new opioids available makes administration more difficult.

Strongly Agree Agree Neither agree nor disagree Disagree Strongly Disagree

Q2. I require more knowledge about opioids (eg, morphine) compared to other medications (eg, blood pressure medications or insulin) in order to give them safely.

Strongly Agree Agree Neither agree nor disagree Disagree Strongly Disagree

Q3. When giving opioids (eg, morphine) I need to monitor patients more closely in comparison to giving other medications (eg, blood pressure medications or insulin).

Strongly Agree Agree Neither agree nor disagree Disagree Strongly Disagree 
Q4. Opioids (eg, morphine) scare me which means I am less likely to want to administer them.

Strongly Agree Agree Neither agree nor disagree Disagree Strongly Disagree

Q5. When giving opioids (eg, morphine) I am afraid of overdose.

Strongly Agree Agree Neither agree nor disagree Disagree Strongly Disagree

Q6. Prescribing errors are a common barrier to nurses administering opioids (eg, morphine).

Strongly Agree Agree Neither agree nor disagree Disagree Strongly Disagree

Q7. Opioids (eg, morphine) are dangerous because they are controlled drugs and require double signing.

Strongly Agree Agree Neither agree nor disagree Disagree Strongly Disagree

Q8. Nurses often associate giving opioids (eg, morphine) with helping patients to die.

Strongly Agree Agree Neither agree nor disagree Disagree Strongly Disagree

Q9. Familiarity with an opioid (eg, morphine) gives me more confidence when administering this opioid.

Strongly Agree Agree Neither agree nor disagree Disagree Strongly Disagree

Q10. When giving opioids (eg, morphine) I am constantly aware of side effects.

Strongly Agree Agree Neither agree nor disagree Disagree Strongly Disagree

Q11. Nurses associate opioids (eg, morphine) with drug abuse.

Strongly Agree Agree Neither agree nor disagree Disagree Strongly Disagree

Q12. When using opioids (eg, morphine) I don't want to make mistakes because I am afraid of criminal investigations.

Strongly Agree Agree Neither agree nor disagree Disagree Strongly Disagree

Q13. When administering opioids (eg, morphine) I am more concerned about patients with a history of drug abuse (IVDU).

Strongly Agree Agree Neither agree nor disagree Disagree $\quad$ Strongly Disagree

Q14. I need to trust the prescribing doctor in order to be comfortable with giving an opioid (eg, morphine).

Strongly Agree Agree Neither agree nor disagree Disagree Strongly Disagree

Thank you for taking the time to complete this questionnaire.

Figure SI Questionnaire.

Journal of Pain Research

Publish your work in this journal

The Journal of Pain Research is an international, peer reviewed, open access, online journal that welcomes laboratory and clinical findings in the fields of pain research and the prevention and management of pain. Original research, reviews, symposium reports, hypothesis formation and commentaries are all considered for publication.

The manuscript management system is completely online and includes a very quick and fair peer-review system, which is all easy to use. Visit http://www.dovepress.com/testimonials.php to read real quotes from published authors. 